\title{
A case of DIPNECH presenting as usual interstitial pneumonia
}

\author{
The authors declare no financial disclosure
}

\begin{abstract}
Diffuse idiopathic pulmonary neuroendocrine cell hyperplasia (DIPNECH) is a rare disease that is classically described as presenting with cough, dyspnea, and wheezing in non-smoker middle aged females. Pulmonary function tests commonly demonstrate an obstructive pattern and CT of chest usually reveals diffuse air trapping with mosaic pattern. We present a case of patient with DIPNECH manifesting with restrictive pattern and as usual interstitial pneumonia on imaging.
\end{abstract}

Key words: DIPNECH; neuroendocrine cells/pathology; lung diseases/pathology

Pneumonol Alergol Pol 2016; 84: 174-177

\section{Introduction}

Diffuse idiopathic pulmonary neuroendocrine cell hyperplasia (DIPNECH) is a rare disorder with much knowledge about its diagnosis and treatment based on reported cases. We present a case of a patient who presented with exertional dyspnea and evidence of usual interstitial pneumonia on imaging. We briefly describe the diagnosis and therapeutic options for DIPNECH described in literature.

\section{Case presentation}

A 66-year-old female non-smoker presented to clinic with progressively worsening shortness of breath on exertion and non-productive cough of several months' duration. She denied orthopnea, paroxysmal nocturnal dyspnea, or edema. Her comorbidities included hypertension and coronary artery disease. She did not report any significant environmental exposures or family history of lung diseases. She did not have any history of rheumatic or collagen vascular disease. Physical exami- nation was significant for bilateral wheezing and fine end inspiratory crackles at the lung bases. Pulmonary function tests (PFTs) demonstrated a restrictive pattern with reduced $\mathrm{FEV}_{1}$ \& FVC (42\% and $40 \%$ of predicted respectively) but preserved $\mathrm{FEV}_{1} / \mathrm{FVC}$ ratio; decreased TLC (2.76 l [54\% of predicted]); and reduced DLCO (47\%). Chest X-ray demonstrated extensively increased interstitial markings with fibronodular character. High resolution computed tomography (HRCT) of chest showed diffuse sub-pleural reticulation and honeycombing involving both upper and lower lobes (Figs 1A, B). The results of the laboratory tests ANA, ESR, CRP, CBC, Serum IgE including bronchoalveolar lavage (BAL) components were in normal range. Microscopic examination of the lung biopsy (the wedge resection sample performed via video assisted thoracoscopic surgery [VATS]) showed interstitial fibrosis and focal bronchiolar neuroendocrine cell hyperplasia with multiple carcinoid tumorlets $(<5 \mathrm{~mm})$. The positive immunoreaction with antibody against synaptophysin confirmed neuroendocrine nature of these cells $(<5 \mathrm{~mm})$ (Figs 2A, B). Her serum

Address for correspondence: Kshitij Chatterjee, University of Arkansas for Medical Sciences, e-mail: kchatterjee@uams.edu

10.5603/PiAP.2016.0020

Received: 14.02.2016

Copyright (C) 2015 PTChP

ISSN 0867-7077 

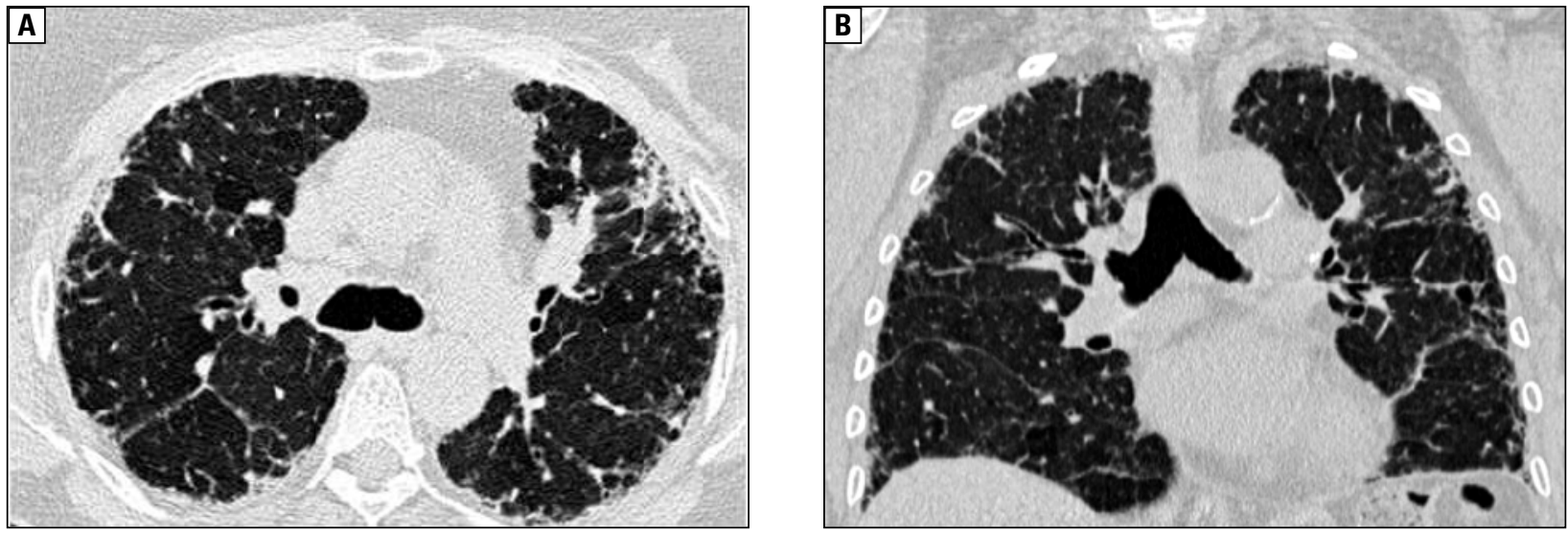

Figure 1A, B. High resolution Computed Tomography (HRCT) of chest demonstrating diffuse interstitial fibrosis and sub-pleural honeycombing involving both upper and lower lobes
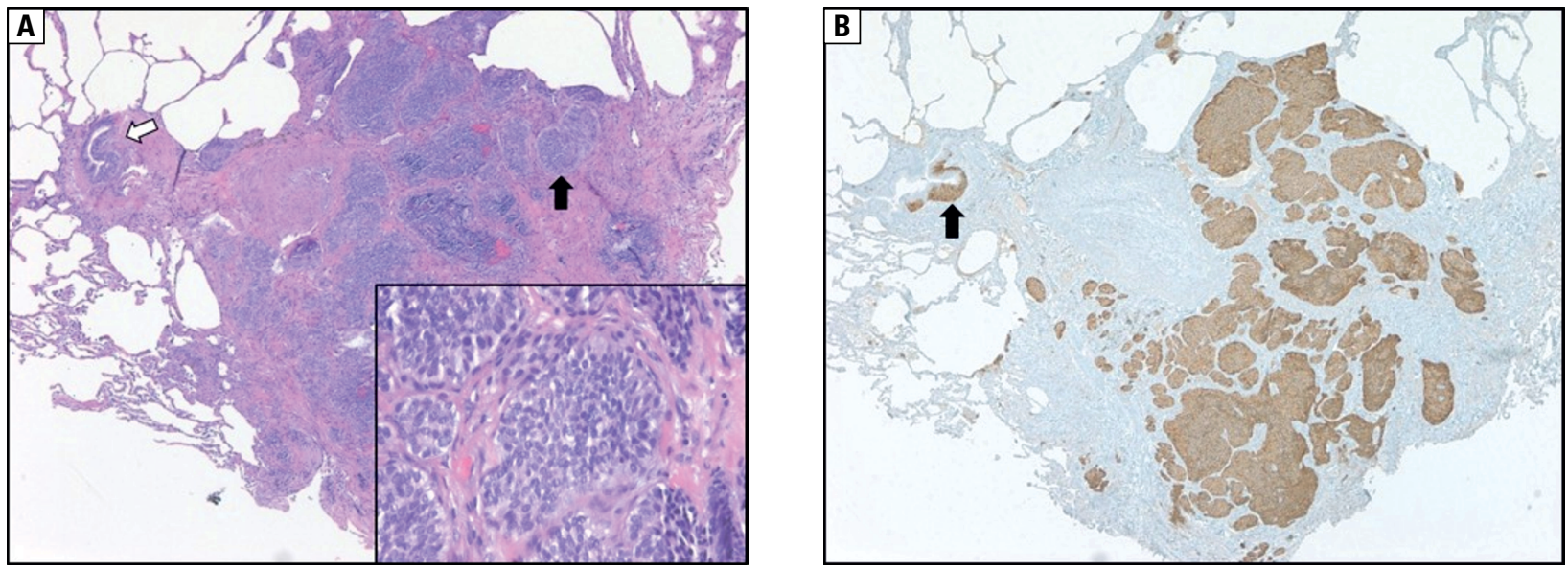

Figure 2. A - tests of polygonal cells (black arrow) with oval nuclei and granular chromatin, measuring $2 \mathrm{~mm}$ in maximum dimension, embedded in a background of interstitial fibrosis. The bronchiole is found to have an increased number of subepithelial cells (white arrow) with oval, granular nuclei consistent with neuroendocrine features. (H\&E, primary magnification $4 \times$ ). Inset High power view of nest of polygonal cells with granular, "salt and pepper" chromatin ( $\mathrm{H \& E}$, primary magnification $40 \times$ ). B - positive immunohistochemical staining for synaptophysin highlights the neuroendocrine nature of the nested cells and hyperplastic subepithelial cells, which are restricted to the basement membrane (black arrow) (primary magnification $4 \times 1$

chromogranin A (CgA) was elevated at $276 \mathrm{ng} / \mathrm{ml}$ (normal range: $0-95 \mathrm{ng} / \mathrm{ml}$ ). Somatostatin receptor scintigraphy (octreotide scan) revealed diffuse, intense radiotracer activity in bilateral lungs (Fig. 3). The listed above evidence allowed the diagnosis of DIPNECH with associated tumorlets. The patient was treated with corticosteroids and azithromycin, however, without apparent improvement. Given the presence of carcinoid tumorlets and an avid diffuse uptake of radiotracer on octreotide scan, she was started on long acting octreotide therapy. After a few weeks of therapy, she experienced better control of her symptoms and improvement in her functional status. CT performed after one year showed stabilization of the pulmonary changes.

\section{Discussion}

Pulmonary neuroendocrine cells (PNEC) are specialized epithelial cells located throughout the respiratory tract. These cells undergo reactive hyperplasia in chronic smokers or in response to hypoxia in patients with underlying lung disease or those living at high altitude [1]. The presence of diffuse proliferation of PNEC without the aforementioned risk factors is suggestive of DIPNECH. It is more prevalent in females (92\%) with a mean age at presentation of 58 years [2]. Based on a review of literature by Nassar et al., the most common presenting symptoms reported are cough (71\%), dyspnea (63\%), and wheezing (25\%) [2]. The most common finding 


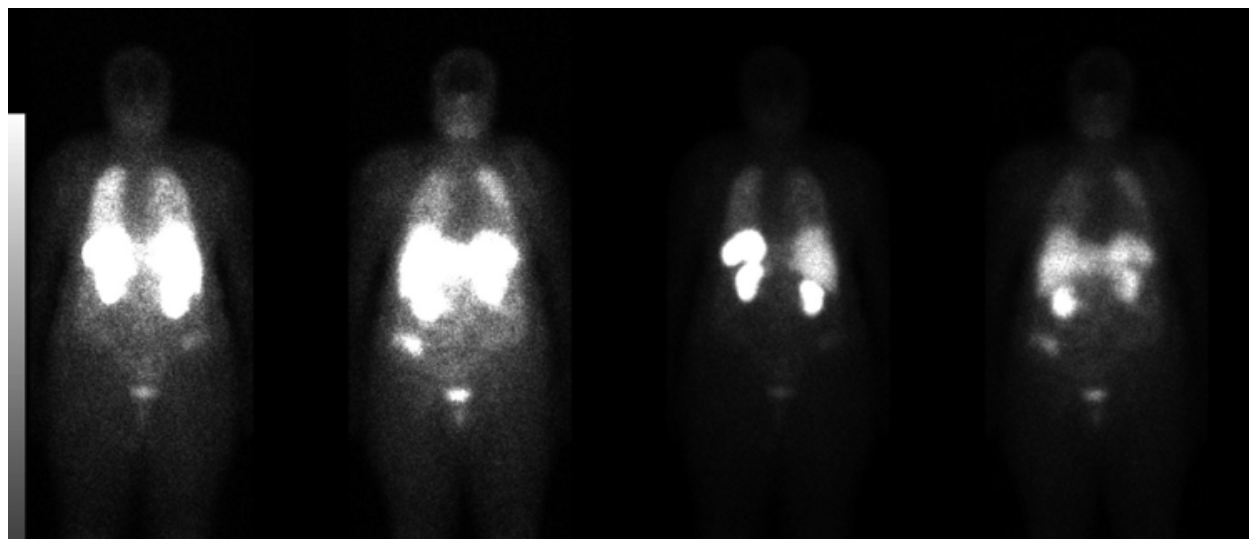

Figure 3. Somatostatin receptor scintigraphy significant for diffuse, intense radiotracer activity in bilateral lungs

on PFTs were an irreversible obstructive defect (54-78\% of patients), however, mixed (17\%), and restrictive pattern (13\%) were also noted [2]. The presence of different types of PFT patterns can be explained by the underlying pathophysiology. Constrictive bronchiolitis resulting in luminal narrowing and mural scarring can be associated with DIPNECH and give rise to obstructive symptoms. The secretion of peptides by the same hyperplastic PNEC can also lead to interstitial fibrosis and restrictive physiology as well $[3,4]$.

In the series of patients with DIPNECH reported by Aguayo et al; all but one patient had diffuse reticulonodular opacities on chest X-ray (CXR) [1]. Single or multiple pulmonary nodules were the most common CT finding in patients with DIPNECH [2]. Mosaic perfusion is also a very commonly reported CT finding in DIPNECH that results from vasoconstriction in the areas of small airway obstruction [3]. Somatostatin receptor scintigraphy, also known as octreotide scan, is a very valuable investigation for detection and staging of neuroendocrine tumors [5]. It is based on the principle that neuroendocrine tumors have an over-expression of somatostatin receptors. The commonly used agent for octreotide scan is ${ }^{111} \mathrm{In}$ -pentetreotide which shows intense uptake by the tumor cells and the hyperplastic neuroendocrine cells in DIPNECH [6]. The role of octreotide scan in DIPNECH extends beyond detection and can help guide treatment strategies. It is postulated that the intensity of uptake of the radiotracer, which represents the density of somatostatin receptors, might predict the chances of therapeutic success with somatostatin analogues [7]. Our patient, who had an intense uptake of radiotracer in bilateral lungs responded very well to therapy with octreotide with resultant improvement in her functional status and decreased oxygen demand.
The microscopic description of DIPNECH includes the presence of widespread hyperplasia of the pulmonary neuroendocrine cells confined to the bronchiolar epithelium. It is recognized as a preneoplastic lesion and patients with DIPNECH might have associated tumorlets $(<5 \mathrm{~mm})$ or carcinoid tumors $(>5 \mathrm{~mm}$ ). The latter two exhibit local invasion and carcinoid can be metastatic as well. The prevalence of DIPNECH in patients with resected carcinoid tumors of lung was reported to be $5.4 \%$ [8]. A large representative lung biopsy sample is required to confirm the diagnosis of DIPNECH with the use of ancillary immunohistochemical staining for neuroendocrine markers like chromogranin, neuron specific enolase, and synaptophysin. Gorshtein et al. performed a retrospective analysis of 11 patients with DIPNECH including their biochemical analysis and found that serum $\mathrm{CgA}$ was elevated in 6 out of 11 patients, however, 24-hour urine excretion of 5-hydroxyindoleaceticacid (5-HIAA) was normal in all patients [6].

DIPNECH is classified as a preinvasive precursor to pulmonary carcinoid and tumorlets. However, most patients with DIPNECH have a stable clinical course [9]. Treatment with short acting beta agonists, inhaled corticosteroids, and long acting beta agonists provide symptomatic relief. Systemic steroids have been shown to be beneficial in some cases by possibly reducing the inflammatory response induced by the secretions of PNEC [2]. Azithromycin has also shown benefit in stabilizing the clinical course in some cases, possibly due to its immunomodulatory effects [10]. Therapy with somatostatin analogues (SSA) has been shown to improve the symptoms in some patients with DIPNECH, possibly by reducing hyperplasia and fibrosis by suppression of PNEC peptide production [11, 12]. 
Alteration in activity of mammalian target of rapamycin (mTOR) has been found in many tumors, including neuroendocrine tumors [13]. Rossi et al. tested phosphorylated-mTOR (p-mTOR) and its major target, ribosomal p70S6-kinase (p70S6K) in four patients with DIPNECH associated with carcinoids and tumorlets [14]. They found a diffuse expression of p-mTOR and p70S6K in all neuroendocrine lesions. Sirolimus, an inhibitor of (mTOR) was successfully used in a patient with DIPNECH and carcinoid tumorlets recently [15]. Future studies are required to evaluate the role of mTOR inhibitors as a specific therapy for DIPNECH and associated pulmonary neuroendocrine tumors.

\section{Conclusion}

Diffuse idiopathic pulmonary neuroendocrine cell hyperplasia can present as a syndrome of obstructive and/or interstitial lung disease. DIPNECH should be considered as a differential for subacute/chronic pulmonary disease in nonsmoker middle-aged females presenting with progressive dyspnea, wheezing and evidence of mosaic attenuation, pulmonary nodules, or interstitial changes on CT. It is also commonly associated with tumorlets or carcinoid tumors. Corticosteroids, azithromycin, and somatostatin analogues have been shown to be of benefit in reported cases of DIPNECH. Once the diagnosis is confirmed by biopsy, Octreotide scan can be a valuable tool for guidance of therapy and evaluating response.

\section{Conflict of interest}

The authors declare no conflict of interest.

\section{References}

1. Aguayo SM, Miller YE, Waldron JA et al. Brief report: idiopathic diffuse hyperplasia of pulmonary neuroendocrine cells and airways disease. N Engl J Med 1992; 327: 1285-1288. doi: 10.1056/NEJM199210293271806.

2. Nassar AA, Jaroszewski DE, Helmers RA, Colby T V, Patel BM, Mookadam F. Diffuse idiopathic pulmonary neuroendocrine cell hyperplasia: a systematic overview. Am J Respir Crit Care Med 2011; 184: 8-16. doi: 10.1164/rccm.201010-1685PP.

3. Chassagnon G, Favelle O, Marchand-Adam S, Muret A De, Revel MP. DIPNECH: when to suggest this diagnosis on CT. Clin Radiol 2015; 70: 317-325. doi: 10.1016/j.crad.2014.10.012.

4. Degan S, Lopez GY, Kevill K, Sunday ME. Gastrin-releasing peptide, immune responses, and lung disease. Ann N Y Acad Sci 2008; 1144: 136-147. doi: 10.1196/annals.1418.022.

5. Balon HR, Brown TLY, Goldsmith SJ et al. The SNM practice guideline for somatostatin receptor scintigraphy 2.0. J Nucl Med Technol 2011; 39: 317-324. doi:10.2967/ /jnmt.111.098277.

6. Gorshtein A, Gross DJ, Barak D et al. Diffuse idiopathic pulmonary neuroendocrine cell hyperplasia and the associated lung neuroendocrine tumors: clinical experience with a rare entity. Cancer 2012; 118: 612-619. doi: 10.1002/cncr.26200.

7. Rossi G, Cavazza A, Graziano P, Papotti M. mTOR/p70S6K in diffuse idiopathic pulmonary neuroendocrine cell hyperplasia. Am J Respir Crit Care Med 2012; 185: 341; author reply 341-342.

8. Ruffini E, Bongiovanni M, Cavallo A et al. The significance of associated pre-invasive lesions in patients resected for primary lung neoplasms. Eur J Cardiothorac Surg 2004; 26: 165-172. doi: 10.1016/j.ejcts.2004.03.044.

9. Falkenstern-Ge RF, Kimmich M, Friedel G, Tannapfel A, Neumann V, Kohlhaufl M. Diffuse idiopathic pulmonary neuroendocrine cell hyperplasia. 7-year follow-up of a rare clinicopathologic syndrome. J Cancer Res Clin Oncol 2011; 137: 1495-1498. doi: 0.1007/s00432-011-1015-z.

10. Dhungana S, McCormick J. Successful Treatment of DIPNECH With Azithromycin. CHEST J 2014; 146 (4_MeetingAbstracts): $80 \mathrm{~A}$.

11. Carr LL, Chung JH, Duarte Achcar R et al. The clinical course of diffuse idiopathic pulmonary neuroendocrine cell hyperplasia. CHEST J 2015; 147: 415-422. doi:10.1378/chest.14-0711.

12. Elshafie O, Jayakrishnan B, Al-Busaidi M, Al-Riyami B, Hussein S, Woodhouse N. Chromogranin-A levels and refractory bronchospasm in interstitial lung disease: a preliminary report on the favourable response to octreotide. Br J Med Med Res 2015; 7: 705-710. doi: 10.9734/BJMMR/2015/15655.

13. Righi L, Volante M, Rapa I et al. Mammalian target of rapamycin signaling activation patterns in neuroendocrine tumors of the lung. Endocr Relat Cancer 2010; 17: 977-987. doi: 10.1677/ ERC-10-0157.

14. Rossi G, Cavazza A, Graziano P, Papotti M. mTOR/p70S6K in diffuse idiopathic pulmonary neuroendocrine cell hyperplasia. Am J Respir Crit Care Med 2012; 185: 341-341. doi: 10.1164/ajrccm.185.3.341.

15. Mourad M, Hamblin MJ. Sirolimus for the Treatment of Diffuse Idiopathic Pulmonary Neuroendocrine Cell Hyperplasia (DIPNECH) (ATS Journals). Am Thorac Soc Int Conf Meet Abstr 2015. A1501-A1501.doi:10.1164/ajrccm-conference.2015.191.1_ MeetingAbstracts.A1501 